\title{
Mathematical Modelling and Optimal Control of Anthracnose
}

\author{
David Fotsa $^{1}$, Elvis Houpa ${ }^{2}$, David Bekolle ${ }^{2}$, Christopher Thron ${ }^{3}$ and Michel Ndoumbe ${ }^{4}$ \\ ${ }^{1}$ ENSAI, The University of Ngaoundere, Email: mjdavidfotsa@gmail.com \\ ${ }^{2}$ Faculty of Science, The University of Ngaoundere \\ Email: e-houpa@yahoo.com, bekolle@yahoo.fr \\ ${ }^{3}$ Texas A\&M University, Central Texas \\ Email:thron@ct.tamus.edu \\ ${ }^{4}$ IRAD, Cameroon \\ Email: michel.ndoumbe@yahoo.com
}

Received: 19 November 2013, accepted: 16 April 2014, published: 28 May 2014

\begin{abstract}
In this paper we propose two nonlinear models for the control of anthracnose disease. The first is an ordinary differential equation (ODE) model which represents the within-host evolution of the disease. The second includes spatial diffusion of the disease in a bounded domain. We demonstrate the well-posedness of those models by verifying the existence of solutions for given initial conditions and positive invariance of the positive cone. By considering a quadratic cost functional and applying a maximum principle, we construct a feedback optimal control for the ODE model which is evaluated through numerical simulations with the scientific software Scilabß. For the diffusion model we establish under some conditions the existence of a unique optimal control with respect to a generalized version of the cost functional mentioned above. We also provide a characterization for this optimal control.
\end{abstract}

KeyWords- Anthracnose modelling, nonlinear systems, optimal control. AMS Classification49J20, 49J15, 92D30, 92D40.

\section{INTRODUCTION}

Anthracnose is a phytopathology which attacks a wide range of commercial crops, including almond, mango, banana, blueberry, cherry, citrus, coffee, hevea and strawberry. The disease has been identified in such diverse areas as Ceylon (1923), Guadeloupe (1925), Sumatra (1929), Indochina (1930), Costa Rica (1931), Malaysia (1932), Java (1933), Madagascar (1934), Cameroon (1934), Colombia (1940), Salvador (1944), Brazil (1946), Nyassaland (1949), New Caledonia (1954), and Arabia (1956) [5]. Anthracnose can affect various parts of the plant, including leaves, fruits, twigs and roots. Possible symptoms include defoliation, fruit rot, fruit fall and crown root rot, which can occur before or after harvest depending on both pathogen and host [5], [28].

The Anthracnose pathogen belongs to the Colletotrichum species (acutatum, capsici, gloeosporioides, kahawae, lindemuthianum, musae, ...). Colletotrichum is an ascomycete fungus. It can 


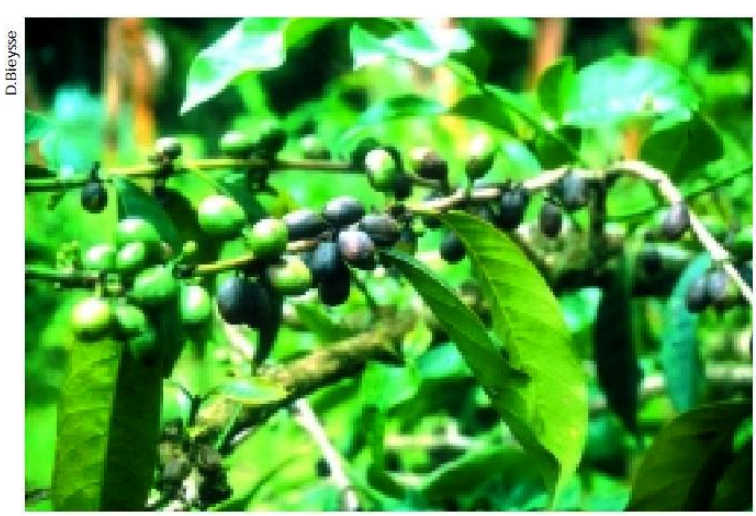

Fig. 1. Symptoms of Coffee Berry Disease (CBD) [4]

reproduce either asexually or sexually, but sexual reproduction is rare in nature [28]. Favourable growth conditions occur particularly in tropical zones. Rainfall, wetness and altitude are all conducive to sporulation and conidia spreading [18], [23]. Sources of inoculum are thought to be leaves, buds and mummified fruits.

\section{A. Anthracnose pathosystem}

The process of infection by Colletotrichum species can usually be divided into at least seven steps, depending on various factors including growth conditions, host tissues and involved species. Conidia deposited on the host attach themselve on its surface. The conidia germinate after 12-48 hours, and appressoria are produced [5], [15]. Severals studies on infection chronology show that appressoria production can occur between 3-48 hours following germination under favourable conditions of wetness and temperature [18], [28]. The pathogen then penetrates the plant epidermis, invades plant tissues, produces acevuli and finally sporulates. The penetration of plant epidermis is enabled by a narrow penetration peg that emerges from the appressorium base [7]. In some marginal cases penetration occurs through plant tissues' stomata or wounds. Once the cuticle is crossed, two infection strategies can be distiguished: intracellular hemibiotrophy and subcuticular intramural necrotrophy, as shown in Figure 2. Invasion of the host is led through formation of hyphae which narrow as the infection progresses. Colletotrichum produce enzymes that degrade carbohydrates, dissolve cell walls, and hydrolyze cuticle. Some of those enzymes are polyglacturonases, pectin lyases and proteases. Some hosts may employ various biochemical strategies to counter the pathogen. For example, the peel of unripe avocados has been found in vitro to contain a preformed antifungal diene (cis, cis-1-acetoxy2-hydroxy-4-oxo-heneicosa-12, 15-diene) that inhibits the growth of Colletotrichum gloeosporioides when present above a certain concentration [28].

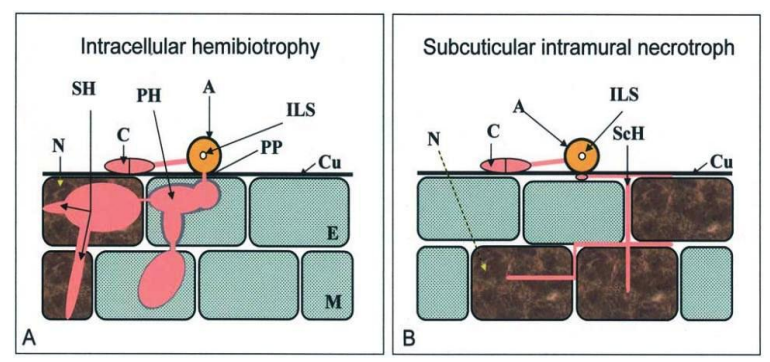

Fig. 2. Infection strategies. (A)=Apressorium $(\mathrm{C})=$ Conidium $\quad-\quad(\mathrm{Cu})=$ Cuticle $\quad-\quad(\mathrm{E})=$ Epidermal (ILS)=Internal Light Spot $\quad-\quad(\mathrm{M})=$ Mesophyl cell $(\mathrm{N})=$ Necrotrophic $-(\mathrm{PH})=$ Primary Hyphae $-(\mathrm{PP})=$ Penetration Peg - $(\mathrm{ScH})=$ Subcuticular and Intramural Hyphae $(\mathrm{SH})=$ Secondary Hyphae [28]

\section{B. Models in the literature}

Most previous mathematical studies on Colletotrichum-host pathosystem have focused on forecasting disease onset based on environmental factors affecting host sensitivity. DANNEBERGER et al. in [9] have developed a forecasting model for the annual bluegrass anthracnose severity index, using weather elements such as temperature and wetness. Their model is a quadratic regression

$$
A S I=a_{0}+a_{0,1} W+a_{1,0} T+a_{1,1} T \times W+a_{0,2} T^{2}+a_{2,0} W^{2}
$$

where $A S I$ is the anthracnose severity index, $T$ is the daily average temperature and $W$ is the average number of hours of leaves' wetness per 
day. DODD et al. in [10] have studied the relationship between temperature $(T)$, relative humidity $(H)$, incubation period $(t)$ and the percentage $(p)$ of conidia of Colletotrichum gloeosporioides producing pigmented appressoria on one month old mangoes. They used the following logistic model:

$$
\ln (p /(1-p))=a_{0}+a_{0,1} H+a_{1,0} T+a_{0,2} H^{2}+a_{2,0} T^{2}+b \ln (t)
$$

DUTHIE in [12] examines the parasite's response $(R)$ to the combined effects of temperature $(T)$ and wetness duration $(W)$. That response could be the rate of germination, infection efficiency, latent period, lesion density, disease incidence or disease severity. Several models are discussed, the two principal being

$$
R(T, W)=f(T)\left[1-\exp \left(-[b(W-c)]^{d}\right)\right]
$$

and

$$
R(T, W)=a\left[1-\exp \left(-[f(T)(W-c)]^{d}\right)\right]
$$

where

$$
f(T)=\frac{e(1+h) h^{\frac{h}{1+h}}}{(1+\exp (g[T-f]))} \exp \left(\frac{g[T-f]}{1+h}\right)
$$

and

$$
a>0, b>0, W \geq c \geq 0, d>0, e>0, f \geq 0, g>0, h>0 .
$$

MOUEN et al. attempt in [17] to develop a spatiotemporal model to analyse infection behaviour with respect to the time, and identify potential foci for disease inoculum. Logistic regression and kriging tools are used used. In addition to these references, there are several other statistical models in literature [12], [17], [18], [19], [20], [21], [28].

\section{Controlling anthracnose}

There are many approaches to controlling anthracnose diseases. The genetic approach involves selection or synthesis of more resistant cultivars [3], [4], [5], [14], [27]. Several studies have demonstrated the impact of cultivational practices on disease dynamics [19], [20], [21], [28]. Other tactics may be used to reduce predisposition and enhance resistance, such as pruning old infected twigs, removing mummified fruits, and shading [5]. Biological control uses microorganisms or biological substrates which interact with pathogen or induce resistance in the host [11]. Finally there is chemical control, which requires the periodic application of antifungal compounds [5], [22], [24]. This seems to be the most reliable method, though relatively expensive. The best control policy should schedule different approaches to optimize quality, quantity and cost of production. Note that inadequate application of treatments could induce resistance in the pathogen [26].

\section{Organization of the paper}

The remainder of this paper is organized as follows. In section II we propose and study a within-host model of anthracnose. We present that model and give parameters meaning in subsection II-A. Throughout subsection II-B we establish the well-posedness of the within-host model both in mathematical and epidemiological senses. The optimal control of the model is surveyed in subsection II-C and numerical simulations are performed in the last subsection II-D. We make a similar study on a spatial version of the model includind a diffusion term in section III. That last model is presented in subsection III-A. Studies on its well-posedness and its optimal control are made respectively in subsections III-B and III-C. Finally, in section IV we discuss our modelling and some realistic generalizations which could be added to the model.

\section{A WITHIN-HOST MODEL}

\section{A. Specification of the within-host model}

The detrimental effects of Colletotrichum infection on fruit growth are closely related to its life cycle. It is mathematically convenient to express these effects in terms of the effective inhibition rate (denoted by $\theta$ ), which is a continuous function of time. The effective inhibition rate is defined such that the maximum attainable fruit volume is reduced by a factor $1-\theta$ if current infection conditions are maintained. In addition to $\theta$, the other time-dependent variables in the model are 
host fruit total volume and infected volume, denoted by $v$ and $v_{r}$ respectively. We have on the set $S=\mathbb{R}_{+} \backslash\{1\} \times \mathbb{R}_{+}^{*} \times \mathbb{R}_{+}$the following equations for the time-evolution of the variables $\left(\theta, v, v_{r}\right)$ :

$$
\left\{\begin{array}{l}
d \theta / d t=\alpha(t, \theta)\left(1-\theta /\left(1-\theta_{1} u(t)\right)\right) \\
d v / d t=\beta(t, \theta)\left(1-v \theta_{2} /\left((1-\theta) \eta(t) v_{\max }\right)\right) \\
d v_{r} / d t=\gamma(t, \theta)\left(1-v_{r} / v\right)
\end{array}\right.
$$

The parameters in (1) have the following practical interpretations:

- $\alpha, \beta, \gamma$ characterize the effects of environmental and climatic conditions on the rate of change of inhibition rate, fruit volume, and infected fruit volume respectively. These are all positive functions of the time $t$ and inhibition rate $\theta$.

- $\gamma$ is an increasing function with respect to $\theta$ and satisfies $\gamma(t, 0)=0, \forall t \geq 0$.

- $u$ is a measurable control parameter which takes values in the set $[0,1]$.

- $1-\theta_{1} \in[0,1]$ is the inhibition rate corresponding to epidermis penetration. Once the epidermis has been penetrated, the inhibition rate cannot fall below this value, even under maximum control effort. In the absence of control effort $(u(t)=0)$, the inhibition rate increases towards 1 .

- $\eta$ is a function of time that characterizes the effects of environmental and climatic conditions on the maximum fruit volume. Its range is the interval $\left.] 0, \theta_{2}\right]$.

- $v_{\max }$ represents the maximum size of the fruit.

- $1-\theta_{2} \in[0,1]$ is the value of inhibition rate $\theta$ that corresponds to a limiting fruit volume of $\eta v_{\max }$. According to the second equation in (1), the limiting volume size is $\eta v_{\max }(1-\theta) / \theta_{2} \leq v_{\max }$. When the volume is less than this value, it increases (but never passes the limiting value); while if the volume exceeds this value, then it decreases. This limiting value for $v$ is less than $\eta v_{\max }$ when $\theta>1-\theta_{2}$ (note $\left.\eta \leq \theta_{2} \leq 1\right)$.
Note that equations (1) are constructed so that $v \leq v_{\max }$ and $v_{r} \leq v$ as long as initial conditions satisfy these inequailities.

With the definitions

$$
\begin{gathered}
A \equiv\left[\begin{array}{ccc}
\frac{-\alpha(t, \theta)}{\left(1-\theta_{1} u(t)\right)} & 0 & 0 \\
0 & -\frac{\theta_{2} \beta(t, \theta)}{\left((1-\theta) \eta(t) v_{\max }\right)} & 0 \\
0 & 0 & -\frac{\gamma(t, \theta)}{v}
\end{array}\right], \\
B \equiv\left[\begin{array}{lll}
\alpha(t, \theta) & \beta(t, \theta) & \gamma(t, \theta)
\end{array}\right]^{T},
\end{gathered}
$$

and

$$
X \equiv\left[\begin{array}{lll}
\theta & v & v_{r}
\end{array}\right]^{T},
$$

then model (1) can be reformulated as

$$
d X / d t=F(t, X),
$$

where

$$
F(t, X) \equiv A(t, X, u) X+B(t, X) .
$$

As indicated above, model (1) is an exclusively within-host model, and as such does not include the effects of spreading from host to host. (In Section III we propose a diffusion model for between-host spreading.) Such a model has several practical advantages. In practice, monitoring of the spreading of the fungi population is difficult. Furthermore, conidia sources and spreading mechanisms are not well-understood, although the literature generally points to mummified fruits, leaves and bark as sources of inoculum. Instead of controlling the host-to-host transmission, an alternative control method is to slow down the withinhost fungi evolution process. Such an approach enables the use of statistical methods, since large samples of infected hosts may easily be obtained [15].

\section{B. Well-posedness of the within-host model}

In the following discussion, we demonstrate that model (1) is well-posed both mathematically and epidemiologically, under the following standard technical assumptions:

(H1) The control parameter $u$ is measurable.

$(H 2)$ The function $F$ is continuous with respect to the variable $X$. 
(H3) For every compact subset $K \subset S$, there is an integrable map $M_{K}: \mathbb{R}_{+} \rightarrow \mathbb{R}_{+}$such that for every $X$ in $K$ and $t$ in $\mathbb{R}_{+},\|F(t, X)\|_{S} \leq M_{K}(t)$.

Existence of a solution is guaranteed by the following proposition, which follows from a simple application of the Carathéodory theorem.

Proposition 1. For every initial condition $\left(t_{0}, X_{0}\right)$ in $\mathbb{R}_{+} \times S$ there is a function $X\left(t_{0}, X_{0}, t\right)$ which is absolutely continuous and satisfies (2) for almost any time $t \in \mathbb{R}_{+}$.

Uniqueness and smoothness of the solution may be established using the Cauchy-Lipschitz Theorem, based on properties $(H 2)$ and $(H 3)$ of the function $F$.

Next we will etablish positive invariance of the set $S$, and the positive invariance of a bounded subset $B S$. These results are needed to show consistency of the biological interpretation of the solution, as explained below. With the definitions

$$
\begin{gathered}
A_{1} \equiv\left[\begin{array}{ccc}
-\frac{\alpha(t, \theta)}{\left(1-\theta_{1} u(t)\right)} & 0 & 0 \\
0 & -\frac{\theta_{2}}{\left((1-\theta) \eta(t) v_{\max }\right)} & 0 \\
0 & 0 & -\frac{1}{v}
\end{array}\right], \\
A_{2} \equiv\left[\begin{array}{ccc}
\alpha(t, \theta) & 0 & 0 \\
0 & \beta(t, \theta) & 0 \\
0 & 0 & \gamma(t, \theta)
\end{array}\right] \\
B_{1} \equiv\left[\begin{array}{lll}
1 & 1 & 1
\end{array}\right]^{T}
\end{gathered}
$$

and $X$ as defined above, then model (1) can be reformulated as

$$
d X / d t=A_{2}\left(A_{1} X+B_{1}\right) .
$$

Theorem 2. The set $S$ is positively invariant for the system (4).

Proof: A solution to (4) satisfies for every time $t \geq 0$,

$$
\begin{aligned}
X(t) & =\exp \left[\int_{0}^{t} A_{2}(s) \cdot A_{1}(s) d s\right] X(0) \\
& +\int_{0}^{t} \exp \left[\int_{s}^{t} A_{2}(\xi) A_{1}(\xi) d \xi\right] A_{2}(s) B_{1} d s
\end{aligned}
$$

Since $-A_{2}(s) A_{1}(s)$ is a $M$-matrix for every time $s \geq 0, \exp \left[\int_{s}^{t} A_{2}(\xi) A_{1}(\xi) d \xi\right]$ is a positive matrix. Moreover, since $B_{1}$ is nonnegtive, one can conclude that $X$ remain nonnegative when $X(0)$ is taken nonnegative.

Theorem 3. Let $B S$ be the subset of $S$ defined such as

$$
B S=\left\{\left(\theta, v, v_{r}\right) \in \mathbb{R}^{3} ; 0 \leq \theta<1,0<v \leq v_{\max }, 0 \leq v_{r} \leq v\right\}
$$

Then $B S$ is positively invariant for system (4).

Proof: We will show that at each point of the boundary of $B S$, the system (4) returns into $B S$. We prove this by showing that the scalar product of the system time derivative with the normal vector $n$ at each boundary point is nonpositive. It has been already shown that positive orthant is positively invariant. Let

$$
\begin{gathered}
F_{1} \equiv\left\{\left(\theta, v, v_{r}\right) \in B S ; \theta=1\right\} \\
F_{2} \equiv\left\{\left(\theta, v, v_{r}\right) \in B S ; v=v_{\max }\right\} \\
F_{3} \equiv\left\{\left(\theta, v, v_{r}\right) \in B S ; v_{r}=v\right\}
\end{gathered}
$$

For all points on $F_{1}, n$ can be choosen as $(1,0,0)$. Since the control $u$ takes its value in $[0,1]$ which also contains $\theta_{1}, \frac{d \theta}{d t}$ is negative and the result is obtained. For all points on $F_{2}, n$ can be choosen as $(0,1,0)$. Thanks to definition of $\theta_{2}$ and $\eta$, $\frac{d v}{d t}$ is negative and the result is obtained. For all points on $F_{3}, n$ can be choosen as $(0,-1,1)$. $\frac{d v_{r}}{d t}$ is zero, and consequently $F_{3}$ is positively invariant.

The invariance of the set $F_{3}$ is biologically plausible, since once the fruit is totally rotten it remain definitely in that state, the fruit is lost. The set $B S$ is also reasonable for biological reasons: the inhibition rate is bounded, the rotten volume is no larger than the total volume, and fungus attack reduces the size of a mature fruit.

\section{Optimal control of the within-host model}

In this subsection we apply control to model (1), which we repeat here for convenience:

$$
\left\{\begin{array}{l}
d \theta / d t=\alpha(t, \theta)\left(1-\theta /\left(1-\theta_{1} u(t)\right)\right) \\
d v / d t=\beta(t, \theta)\left(1-v \theta_{2} /\left((1-\theta) \eta(t) v_{\max }\right)\right) \\
d v_{r} / d t=\gamma(t, \theta)\left(1-v_{r} / v\right)
\end{array}\right.
$$


For the control problem we focus on the first equation. This equation is controllable in $] 0,1[$ since $\theta$ is continuous and $1-\theta_{1} u(t)$ is an asymptotic threshold which can be set easily. Giving a time $T>0$ (for example the annual production duration) we search for $u$ in $L_{\text {loc }}^{2}\left(\mathbb{R}_{+},[0,1]\right)$ such that the following functional (previously used in [1], [13]) is minimized:

$$
J_{T}(u)=\int_{0}^{T}\left(k u^{2}(t)+\theta^{2}(t)\right) d t+f(\theta(T)),
$$

where $k>0$ can be interpreted as the cost ratio related to the use of control effort $u$. This functional reflects the fact that reducing inhibition rate $\theta$ will lead to increased fruit production (larger volumes with a relatively lower level of infection), while minimizing $u$ will reduce financial and environmental costs. We use the squares of $u$ and $\theta$ in the integrand because this choice facilitates the technical calculations required for minimization.

We note in passing that we could had tried to minimize the more practically relevant expression

$$
\begin{aligned}
& \int_{0}^{T}\left(k u(t)+\theta(t)+\left(v_{\max }-v(t)\right)+v_{r}(t)\right) d t \\
& \quad+\theta(T)+\left(v_{\max }-v(T)\right)+v_{r}(T)
\end{aligned}
$$

However, an exact computation of this functional would require precise expressions for $\alpha, \beta, \gamma, \eta$ in the system (1). As far as the authors know, there is no previous study which gives those parameters. It seemed more advantageous to us to limit the random choice of parameters, so that we could perform representative simulations.

We define the set

$U^{K} \equiv\left\{\begin{array}{c}u \in C([0, T] ;[0,1]) ; \forall t, s \in[0, T], \\ |u(t)-u(s)| \leq K|t-s|\end{array}\right\}$ which is nonempty for every $K \geq 0$.

Theorem 4. Let $K \geq 0$. There is a control $u^{*} \in$ $U^{K}$ which minimizes the cost $J_{T}$.

Proof: Since $J_{T} \geq 0$ it is bounded below. Let the infinimum be $J^{*}$, and let $\left(u_{n}\right)_{n \in \mathbb{N}}$ be a sequence in $U^{K}$ such that $\left(J_{T}\left(u_{n}\right)\right)_{n \in \mathbb{N}}$ converges to $J^{*}$. The definition of $U^{K}$ implies that $\left(u_{n}\right)_{n \in \mathbb{N}}$ is bounded and uniformly equicontinuous on $[0, T]$. By the Ascoli theorem, there is a subsequence $\left(u_{n_{k}}\right)$ which converges to a control $u^{*}$. Since the cost function is continuous with respect to $u$ it follows that $J_{T}\left(u^{*}\right)=J^{*}$.

Theorem 5. Suppose that $\alpha$ depends only on time. If there is an optimal control strategy $u$ which minimizes $J_{T}$, then $u$ is unique and satisfies

$$
u(t)=\left\{\begin{array}{l}
1 \text { when } 27 \alpha \theta_{1}^{2} \theta p \geq 8 k \\
\frac{w_{3}(t)-1}{\theta_{1} w_{3}(t)} \text { when } 27 \alpha \theta_{1}^{2} \theta p<8 k
\end{array}\right.
$$

where $w_{3}(t)$ is the element of $\left[1, \min \left\{3 / 2,1 /\left(1-\theta_{1}\right)\right\}\right]$ which is the nearest to the smallest nonnegative solution of the equation $\alpha \theta_{1}^{2} \theta p w^{3}-2 k w+2 k=0$ and

$$
\left\{\begin{array}{l}
d \theta / d t=\alpha(t, \theta)\left(1-\theta /\left(1-\theta_{1} u(t)\right)\right) \\
d p / d t=\alpha(t) p(t) /\left(1-\theta_{1} u(t)\right)-2 \theta \\
\theta(0)=\theta_{0}, \theta(T)=\theta_{T}, p(T)=f^{\prime}\left(\theta_{T}\right)
\end{array}\right.
$$

Proof: According the maximum principle, minimizing $J_{T}$ is equivalent to minimizing the Hamiltonian functional

$$
\begin{aligned}
H(t, \theta, u) & =k u^{2}(t)+\theta^{2}(t)+f(\theta(T)) \\
& +\alpha(t) p(t)\left(1-\theta_{1} u(t)-\theta\right) /\left(1-\theta_{1} u(t)\right)
\end{aligned}
$$

where the adjoint state $p$ is the solution to the following problem

$$
\left\{\begin{array}{l}
d p / d t=\alpha(t) p /\left(1-\theta_{1} u(t)\right)-2 \theta \\
p(T)=f^{\prime}(\theta(T))
\end{array}\right.
$$

To simplify the expression, let

$$
w \equiv 1 /\left(1-\theta_{1} u\right) \in\left[1,1 /\left(1-\theta_{1} u\right)\right] .
$$

Then the new equivalent functional to minimize is

$$
\begin{gathered}
J_{T}^{1}(w)=\int_{0}^{T}\left(k\left(\frac{w(t)-1}{\theta_{1} w(t)}\right)^{2}+\theta^{2}(t)\right) d t+f(\theta(T)) \\
\partial_{w} H=0 \text { if and only if } \\
\qquad \alpha \theta_{1}^{2} \theta p w^{3}-2 k w+2 k=0 .
\end{gathered}
$$

This equation has a unique nonpositive solution when $27 \alpha \theta_{1}^{2} \theta p \geq 8 k$. It has at least one nonnegative solution when $27 \alpha \theta_{1}^{2} \theta p<8 k$. We can choose $w$ in the following way:

$$
w(t)=\left\{\begin{array}{l}
\frac{1}{1-\theta_{1}} \text { when } 27 \alpha \theta_{1}^{2} \theta p \geq 8 k \\
w_{3}(t) \text { when } 27 \alpha \theta_{1}^{2} \theta p<8 k
\end{array}\right.
$$


where $w_{3}(t)$ is the element of $\left[1, \min \left\{3 / 2,1 /\left(1-\theta_{1}\right)\right\}\right]$ that is the nearest to the smallest nonnegative solution of 10 . It follows from the definition of $w$ in (9) and algebraic rearrangement that the optimal control $u$ is given by (6), where $(p, \theta)$ is a solution to the system (7). The uniqueness of $u$ follows from the uniqueness of the solution of the system (7).

\section{Computer simulations of the controlled within-} host model

We performed simulations in order to demonstrate the practical controllability of the system, For these simulations we used an inhibition pressure of the form

$$
\alpha(t)=a(t-b)^{2}(1-\cos (2 \pi t / c))
$$

with $b$ and $c$ in $[0,1]$. This function reflects the seasonality of empirically-based severity index models found in the literature [9], [10], [12]. The particular values used in the simulation were $a=4, b=0.75, c=0.2$ and $k=1$. We also took $f(\theta(T))=\theta(T)$, so that $p(T)=f^{\prime}(\theta(T))=1$. In this case and the shooting method can be used to numerically estimate the value of $p_{0}$ which produces a solution to (8).

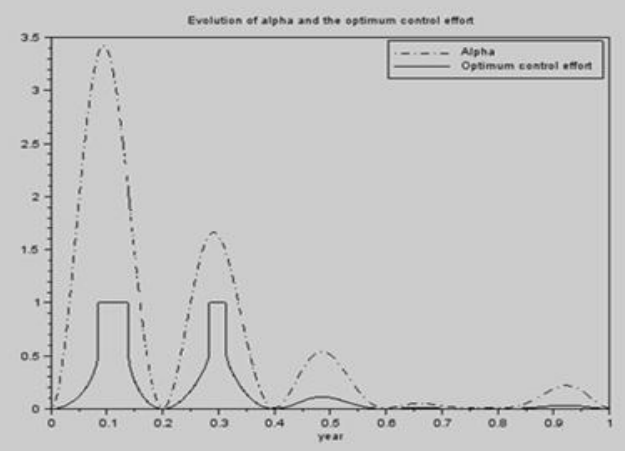

Fig1: Optimum control effort over a one-year period $\theta_{0}=0.2, \theta_{1}=1-0.4$.

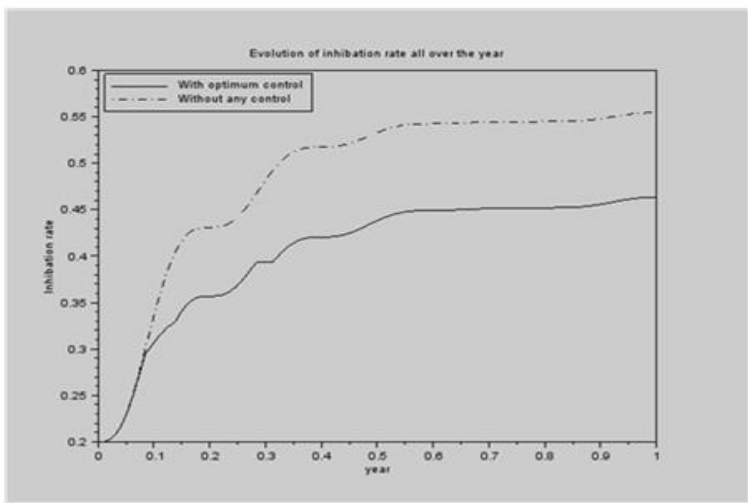

Fig2: Evolution of inhibition rate over a one-year period with $\theta_{0}=0.2, \theta_{1}=1-0.4$.

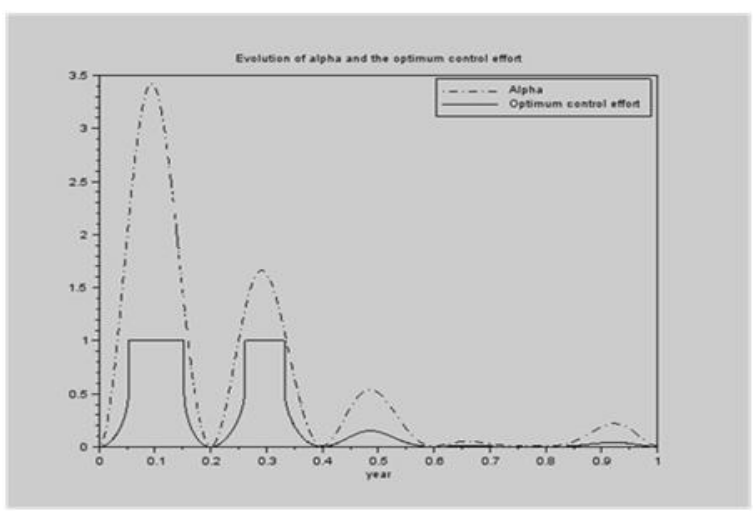

Fig3: Optimum control effort over a one-year period $\theta_{0}=0.5, \theta_{1}=1-0.4$.

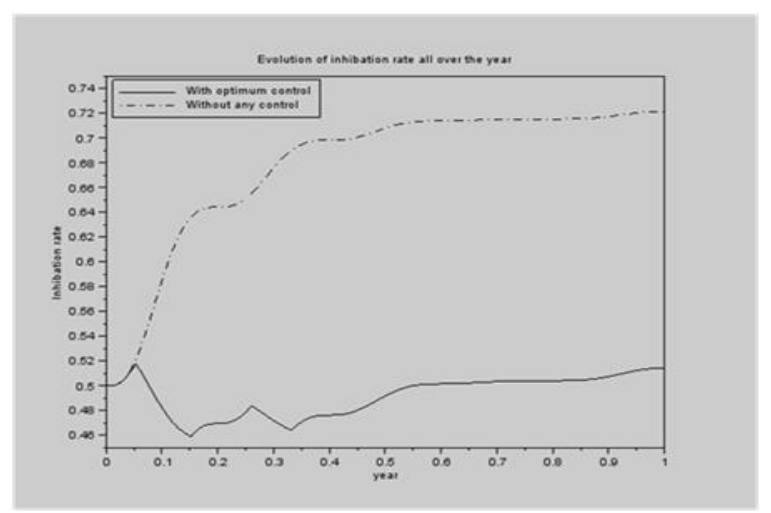

Fig4: Evolution of inhibition rate over a one-year period $\theta_{0}=0.5, \theta_{1}=1-0.4$.

The above figures show how the control strategy adapts itself in response to inhibition pressure represented by $\alpha$. Figures $1-2$ correspond to the case 
of low initial effective inhibition rate $\left(\theta_{0}=0.2\right.$, corresponding to a low initial level of infection), while Figures 3-4 correspond to the case of high initial effective inhibition rate $\left(\theta_{0}=0.5\right)$. The simulations also show in different cases the effectiveness of the optimal strategy as compared to taking no control action. Regardless of whether the initial inhibition rate is above or below the threshold $1-\theta_{1}$, the dynamics are sensitive to the control effort.

\section{A DIFFUSION MODEL}

In this section, we will model the geographical spread of the disease via diffusive factors such as the movement of inoculum through ground water and wind.

\section{A. Specification of the diffusion model}

We include the effect of diffusive factors on the spread of infection by adding a diffusion term to the within-host equation for $\theta$ from system (1). Together with boundary conditions, the model is

$$
\begin{array}{cc}
\partial \theta / \partial t=\alpha(t, x, \theta)\left(1-\theta /\left(1-\theta_{1} u(t, x)\right)\right) & \\
+\operatorname{div}(A(t, x, \theta) \nabla \theta) & \text { on } \mathbb{R}_{+}^{*} \times \Omega, \\
\langle A(t, x, \theta) \nabla \theta, n\rangle=0 & \text { on } \mathbb{R}_{+}^{*} \times \partial \Omega, \\
\theta(0, x)=\theta_{0}(x) \geq 0 & x \in \bar{\Omega},
\end{array}
$$

where $\Omega$ is an open bounded subset of $\mathbb{R}^{3}$ with a continuously differentiable boundary $\partial \Omega$, and $\theta_{1} \in[0,1[$. For a given element $(t, x, \theta), A$ is a $3 \times 3$-matrix $\left(a_{i j}(t, x, \theta)\right)$. The functions $\alpha$ and $a_{i j}$ are assumed to be nonnegative. Since $\theta$ depends on $(t, x)$, the functions $\alpha$ and $a_{i j}$ can be identified with elements of the set $C\left([0, T] ; H^{1}(\Omega)\right)$. The function $u \in C\left([0, T] ; H^{1}(\Omega)\right)$ designates the control, which takes its values in the set $[0,1]$. (12) may be interpreted as a dependence of the flux of inoculum with respect to diffusion factors. In particular, when $A$ is the identity matrix (14) could be interpreted as there is no flux between exterior and interior of the domain $\Omega$.

Practically, time can be subdivided into intervals on which parameters are approximately constant.
We may thus study the system on each interval separately, and assume that all parameters are constant. We also assume that functions $\alpha$ and $A$ do not depend on $\theta$. This leads to the following simplified model,

$$
\begin{aligned}
& \partial \theta / \partial t=\alpha(x)\left(1-\theta /\left(1-\theta_{1} u(x)\right)\right) \\
& +\operatorname{div}(A(x) \nabla \theta) \quad \text { on }] 0, T[\times \Omega \text {, } \\
& \langle A(x) \nabla \theta, n\rangle=0 \quad \text { on }] 0, T[\times \partial \Omega, \\
& \theta(0, x)=\theta_{0}(x) \geq 0 \quad x \in \bar{\Omega},
\end{aligned}
$$

In order to formalize the model, we define the Hilbert space

$$
\left.E=\left\{\theta \in H^{2}(\Omega) ; \theta \text { satisfies } 15\right)\right\}
$$

provided with the inner product

$$
\langle f, g\rangle_{E}=\int_{U}(f g+\langle\nabla f, \nabla g\rangle+\Delta f . \Delta g) d x
$$

Define also the linear unbounded operator $£$ : $D(£)=E \subset L^{2}(\Omega) \rightarrow L^{2}(\Omega)$ as

$$
£ \theta=\frac{\alpha \theta}{1-\theta_{1} u}-\operatorname{div}(A(x) \nabla \theta)
$$

Then equation (14) takes the following form

$$
\partial \theta / \partial t+£ \theta=\alpha .
$$

We also introduce the following condition, which we will use to ensure that the system has realistic solutions:

(H4) There exists a constant $C>0$ such that for almost every $x \in \Omega, A(x)$ is symmetric, positive definite and

$$
\langle v, A(x) v\rangle \geq C\langle v, v\rangle, \forall v
$$

\section{B. Well-posedness of the diffusion model}

We are now ready to prove that our model has been a mathematically and epidemiologically wellposed. In other words, we show that exists a unique solution $0 \leq \theta(t, x) \leq$ to the system 14$)-16$. 
This shall follow from the Hille-Yosida theorem 1 . but first we need the following proposition.

Proposition 6. If $A(x)$ is a positive semidefinite bilinear form for almost every $x \in \Omega$, then the linear operator $£$ is monotone on $E$. Moreover, if $A(x)$ satisfies (H4), then $£$ is maximal.

\section{Proof:}

(ii) To show $£$ is monotone, we let $\theta \in E$ and compute:

$\int_{\Omega} £ \theta \times \theta d x$

$=\int_{\Omega}\left(\alpha \theta^{2} /\left(1-\theta_{1} u\right)\right) d x-\int_{\Omega} \operatorname{div}(A(x) \nabla \theta) \theta d x$

$=\left(1 /\left(1-\theta_{1} u\right)\right)\|\theta \sqrt{\alpha}\|_{L^{2}(\Omega)}^{2}-\int_{\Omega} \operatorname{div}(A(x) \nabla \theta) \theta d x$

$\geq-\int_{\partial \Omega}\langle A(x) \nabla \theta, n(x)\rangle \theta d x+\int_{\Omega}\langle A(x) \nabla \theta, \nabla \theta\rangle d x$ $\geq 0$.

(ii) To show $£$ is maximal, we let $f \in L^{2}(\Omega)$ and seek $\theta \in E$ such that $\theta+£ \theta=f$. Given $\varphi \in E$, we have

$$
\begin{aligned}
& \int_{\Omega}(\theta+£ \theta) \times \varphi d x \\
& =\int_{\Omega} \varphi \theta\left(1+\alpha-\theta_{1} u\right) /\left(1-\theta_{1} u\right) d x \\
& -\int_{\Omega} \operatorname{div}(A(x) \nabla \theta) \varphi d x \\
& =\int_{\Omega} \varphi \theta\left(1+\alpha-\theta_{1} u\right) /\left(1-\theta_{1} u\right) \\
& +\int_{\Omega}\langle A(x) \nabla \theta, \nabla \varphi\rangle d x \\
& -\int_{\partial \Omega}\langle A(x) \nabla \theta, n(x)\rangle \varphi d x \\
& =\int_{\Omega} \varphi \theta\left(1+\alpha-\theta_{1} u\right) /\left(1-\theta_{1} u\right) \\
& +\int_{\Omega}\langle A(x) \nabla \theta, \nabla \varphi\rangle d x \\
& \equiv p(\theta, \varphi),
\end{aligned}
$$

where $p$ is a symmetric continuous and coercive bilinear form on $H^{1}(\Omega)$. The LaxMilgram theorem ${ }^{2}$ implies that there is a unique $\theta \in H^{1}(\Omega)$ such that $\theta+£ \theta=f$.

${ }^{1}$ See [6] p 185.

${ }^{2}$ See [6]
Using regularization methods similar those used in Theorem 9.26 of [6], it follows that $\theta \in E$.

Given that the linear operator $£$ is maximal monotone and $\theta_{0}$ is in $E$, then by the HilleYosida theorem there is a unique function $\theta \in$ $C^{1}\left([0, T] ; L^{2}(\Omega)\right) \bigcap C([0, T] ; E)$ which satisfies $(14)-(16)$, and $\forall(t, x) \in[0, T] \times \Omega$ we have $\theta(t, x)=\left(S_{£}(t) \theta_{0}\right)(x)+\int_{0}^{t}\left(S_{£}(t-s) \alpha\right)(x) d s$,

where $S_{£}(t)$ is the contraction semigroup generated by $-£$.

Now that we have established existence and uniqueness of the solution $\theta$, we now prove that $0 \leq \theta(t, x) \leq 1$ for all $(t, x)$ in the domain. assuming that $A(x)$ satisfies the condition (H4). We define

$$
\begin{aligned}
m & \equiv \inf _{\partial \Omega} \theta_{0} \\
M & \equiv \max \left\{\sup _{\partial \Omega} \theta_{0}, \sup _{\Omega}\left(1-\theta_{1} u\right)\right\}, \\
v & \equiv 1 /\left(1-\theta_{1} u\right) .
\end{aligned}
$$

Note that $M \leq 1$ as long as $\theta_{0} \leq 1$ and $0 \leq \theta_{1} u \leq$ 1.

Let $E_{+}$designate the set of elements in $E$ which are nonnegative almost everywhere on $\bar{\Omega}$. The following theorem gives sufficient conditions under which the solution $\theta$ of (14) - 16 is bounded by $M \leq 1$ and the positive cone $E_{+}$ of $E$ is positively invariant.

Theorem 7. If $A(x) \nabla e^{t v \alpha}=0$ for every $(t, x) \in$ $[0, T] \times \Omega$ then for almost every $x$ in $\Omega$,

$$
m \leq e^{t v \alpha} \theta(t, x), t \in[0, T] .
$$

Moreover if $A(x) \nabla v=0$ for every $(t, x) \in$ $[0, T] \times \Omega$, then

$$
\theta(t, x) \leq M
$$

In particular, (18) and (19) hold when $\alpha$ and $u$ do not depend on the space variable $x$.

Proof: Let $G \in C^{1}(\mathbb{R})$ such that

(i) $G(s)=0, \forall s \leq 0$, and 
(ii) $0<G^{\prime}(s) \leq C, \forall s>0$.

Define

$$
\begin{aligned}
H(s) & \equiv \int_{0}^{s} G(\sigma) d \sigma, \forall s \in \mathbb{R}, \\
\varphi_{1}(t) & \equiv \int_{\Omega} H\left(m-e^{t v \alpha} \theta(t, x)\right) d x, \\
\varphi_{2}(t) & \equiv \int_{\Omega} H\left(e^{t v \alpha}(\theta(t, x)-1 / v)\right) d x .
\end{aligned}
$$

We observe that

$$
\begin{aligned}
& \left.\left.\varphi_{1}, \varphi_{2} \in C([0, T] ; \mathbb{R}) \bigcap C^{1}(] 0, T\right] ; \mathbb{R}\right), \\
& \varphi_{1}, \varphi_{2} \geq 0 \text { on }[0, T] \\
& \varphi_{1}(0)=\varphi_{2}(0)=0 .
\end{aligned}
$$

We may also compute

$$
\begin{aligned}
\varphi_{1}^{\prime}(t) & \\
= & -\int_{\Omega} e^{t v \alpha} G\left(m-e^{t v \alpha} \theta\right)(v \alpha \theta+\partial \theta / \partial t) d x \\
= & -\int_{\Omega} e^{t v \alpha} G\left(m-e^{t v \alpha} \theta\right)(\alpha-£ \theta+v \alpha \theta) d x \\
=- & \int_{\Omega} \alpha G\left(m-e^{t v \alpha} \theta\right) d x \\
& +\int_{\Omega}\left\langle A(x) \nabla \theta, \nabla e^{t v \alpha} G\left(m-e^{t v \alpha} \theta\right)\right\rangle d x \\
=- & \int_{\Omega} \alpha G\left(m-e^{t v \alpha} \theta\right) d x \\
& -\int_{\Omega} e^{2 t v \alpha} G^{\prime}\left(m-e^{t v \alpha} \theta\right)\langle A(x) \nabla \theta, \nabla \theta\rangle d x \\
& +\int_{\Omega}\left(G\left(m-e^{t v \alpha} \theta\right)-e^{t v \alpha} \theta G^{\prime}\left(m-e^{t v \alpha} \theta\right)\right) \\
& \times\left\langle A(x) \nabla \theta, \nabla e^{t v \alpha}\right\rangle d x \\
\leq & 0 .
\end{aligned}
$$

Since $\varphi_{1}^{\prime} \leq 0$ on $\mathbb{R}_{+}^{*}, \varphi_{1}$ is identically zero on $\mathbb{R}_{+}$ and therefore almost everywhere in $\Omega$.

$$
m \leq e^{t v \alpha} \theta(t, x)
$$

If $A(x) \nabla v=0$ for every $(t, x) \in[0, T] \times \Omega$, then $\varphi_{2}^{\prime}(t)$

$$
\begin{aligned}
= & \int_{\Omega} G\left(e^{t v \alpha}(\theta-1 / v)\right)(-\alpha+v \alpha \theta+\partial \theta / \partial t) d x \\
= & \int_{\Omega} e^{t v \alpha} G\left(e^{t v \alpha}(\theta-1 / v)\right)(-£ \theta+v \alpha \theta) d x \\
= & -\int_{\Omega}\left\langle A(x) \nabla \theta, \nabla e^{t v \alpha} G\left(e^{t v \alpha}(\theta-1 / v)\right)\right\rangle d x \\
=- & \int_{\Omega} e^{2 t v \alpha} G^{\prime}\left(e^{t v \alpha}(\theta-1 / v)\right)\langle A(x) \nabla \theta, \nabla \theta\rangle d x \\
& -\int_{\Omega}\left(e^{2 t v \alpha} / v^{2}\right) G^{\prime}\left(e^{t v \alpha}(\theta-1 / v)\right)\langle A(x) \nabla \theta, \nabla v\rangle d x \\
& -\int_{\Omega} G\left(e^{t v \alpha}(\theta-1 / v)\right)\left\langle A(x) \nabla \theta, \nabla e^{t v \alpha}\right\rangle d x \\
& -\int_{\Omega} e^{t v \alpha}(\theta-1 / v) G^{\prime}\left(e^{t v \alpha}(\theta-1 / v)\right) \\
& \times\left\langle A(x) \nabla \theta, \nabla e^{t v \alpha}\right\rangle d x
\end{aligned}
$$

$\leq 0$.

Since $\varphi_{2}^{\prime} \leq 0$ on $\mathbb{R}_{+}^{*}, \varphi_{2}$ is identically zero on $\mathbb{R}_{+}$ and therefore almost everywhere in $\Omega$

$$
\theta(t, x) \leq M \text {. }
$$

The following theorem proves boundedness of $\theta$ under more general conditions.

Theorem 8. Suppose that $v$ and $\alpha v$ are increasing functions $h$ and $g$ of the state $\theta$, and there is $a$ constant $K>0$ such that

$$
a g^{\prime}(\theta) \leq K\left(1+a g^{\prime}(\theta)\right) \exp (a g(\theta)), \forall a \geq 0 \text {. }
$$

Then for every time $t \in[0, T]$ and almost every $x$ in $\Omega$,

$$
m \leq e^{t v \alpha} \theta(t, x)
$$

and

$$
\theta(t, x) \leq M
$$

Proof: Let $G \in C^{1}(\mathbb{R})$ such that

(i) $G(s)=0, \forall s \leq 0$, and

(ii) $K G(s) \leq G^{\prime}(s) \leq C, \forall s>0$.

Using (20) and the fact that operator $A$ is monotone, we have

$$
\begin{aligned}
\langle A(x) \nabla \theta, \nabla v\rangle & =\langle A(x) \nabla \theta, \nabla h(\theta)\rangle \\
& =h^{\prime}(\theta)\langle A(x) \nabla \theta, \nabla \theta\rangle \\
& \geq 0,
\end{aligned}
$$




$$
\begin{aligned}
\left\langle A(x) \nabla \theta, \nabla e^{t w \alpha}\right\rangle & =\langle A(x) \nabla \theta, \nabla \exp (t g(\theta))\rangle \\
& =t g^{\prime}(\theta) \exp (t g(\theta))\langle A(x) \nabla \theta, \nabla \theta\rangle \\
& \geq 0
\end{aligned}
$$

and

$$
\begin{aligned}
& \left\langle A(x) \nabla \theta, \nabla e^{t v \alpha} G\left(m-e^{t v \alpha} \theta\right)\right\rangle \\
= & \langle A(x) \nabla \theta, \nabla \exp (t g(\theta)) G(m-\theta \exp (t g(\theta)))\rangle \\
= & t g^{\prime}(\theta) \exp (t g(\theta)) G(m-\theta \exp (t g(\theta)))\langle A(x) \nabla \theta, \nabla \theta\rangle \\
- & \exp (2 t g(\theta))\left(1+t g^{\prime}(\theta)\right) G^{\prime}(m-\theta \exp (t g(\theta)))\langle A(x) \nabla \theta, \nabla \theta\rangle \\
\leq & \left(t g^{\prime}(\theta)-K\left(1+t g^{\prime}(\theta)\right) \exp (t g(\theta))\right) \exp (t g(\theta)) \\
& \times G(m-\theta \exp (t g(\theta)))\langle A(x) \nabla \theta, \nabla \theta\rangle \\
\leq & 0 .
\end{aligned}
$$

Define

$$
\begin{aligned}
H(s) & \equiv \int_{0}^{s} G(\sigma) d \sigma, \forall s \in \mathbb{R}, \\
\varphi_{1}(t) & \equiv \int_{\Omega} H\left(m-e^{t v \alpha} \theta(t, x)\right) d x, \\
\varphi_{2}(t) & \equiv \int_{\Omega} H\left(e^{t v \alpha}(\theta(t, x)-1 / w)\right) d x .
\end{aligned}
$$

Note that as in Theorem 7 we have

$$
\begin{aligned}
& \left.\left.\varphi_{1}, \varphi_{2} \in C([0, T] ; \mathbb{R}) \bigcap C^{1}(] 0, T\right] ; \mathbb{R}\right), \\
& \varphi_{1}, \varphi_{2} \geq 0 \text { on }[0, T] \\
& \varphi_{1}(0)=\varphi_{2}(0)=0 .
\end{aligned}
$$

As in Theorem 7 we may compute

$$
\begin{aligned}
\varphi_{1}^{\prime}(t) & \\
= & -\int_{\Omega} e^{t v \alpha} G\left(m-e^{t v \alpha} \theta\right)(w \alpha \theta+\partial \theta / \partial t) d x \\
= & -\int_{\Omega} e^{t v \alpha} G\left(m-e^{t v \alpha} \theta\right)(\alpha-£ \theta+v \alpha \theta) d x \\
= & -\int_{\Omega} \alpha G\left(m-e^{t v \alpha} \theta\right) d x \\
& +\int_{\Omega}\left\langle A(x) \nabla \theta, \nabla e^{t v \alpha} G\left(m-e^{t v \alpha} \theta\right)\right\rangle d x \\
\leq & \int_{\Omega}\left\langle A(x) \nabla \theta, \nabla e^{t v \alpha} G\left(m-e^{t v \alpha} \theta\right)\right\rangle d x \\
\leq & 0 .
\end{aligned}
$$

Since $\varphi_{1}^{\prime} \leq 0$ on $\mathbb{R}_{+}^{*}, \varphi_{1}$ is identically null on $[0, T]$ and therefore almost everywhere in $\Omega$

$$
m \leq e^{t v \alpha} \theta(t, x) .
$$

We also have

$$
\begin{aligned}
& \varphi_{2}^{\prime}(t) \\
& =\int_{\Omega} G\left(e^{t v \alpha}(\theta-1 / v)\right)(-\alpha+v \alpha \theta+\partial \theta / \partial t) d x \\
& =\int_{\Omega} e^{t v \alpha} G\left(e^{t v \alpha}(\theta-1 / v)\right)(-£ \theta+v \alpha \theta) d x \\
& =-\int_{\Omega}\left\langle A(x) \nabla \theta, \nabla e^{t v \alpha} G\left(e^{t v \alpha}(\theta-1 / v)\right)\right\rangle d x \\
& =-\int_{\Omega} e^{2 t v \alpha} G^{\prime}\left(e^{t v \alpha}(\theta-1 / v)\right)\langle A(x) \nabla \theta, \nabla \theta\rangle d x \\
& \quad-\int_{\Omega}\left(e^{2 t v \alpha} / v^{2}\right) G^{\prime}\left(e^{t v \alpha}(\theta-1 / v)\right)\langle A(x) \nabla \theta, \nabla v\rangle d x \\
& \quad-\int_{\Omega} G\left(e^{t v \alpha}(\theta-1 / v)\right)\left\langle A(x) \nabla \theta, \nabla e^{t v \alpha}\right\rangle d x \\
& \quad-\int_{\Omega} e^{t v \alpha}(\theta-1 / w) G^{\prime}\left(e^{t v \alpha}(\theta-1 / v)\right) \\
& \quad \times\left\langle A(x) \nabla \theta, \nabla e^{t v \alpha}\right\rangle d x
\end{aligned}
$$$$
\leq 0 \text {. }
$$

Since $\varphi_{2}^{\prime} \leq 0$ on $[0, T] \backslash\{0\}, \varphi_{2}$ is identically null on $[0, T]$ and therefore almost everywhere in $\Omega$

$$
\theta(t, x) \leq M \text {. }
$$

Condition 20 of Theorem 8 is satisfied in particular when $g \geq 0$. Using the same arguments as in the proof of Proposition 6, there is a unique equilibrium $\theta^{*}$, for the system $114-(16) \cdot \theta^{*}$ is asymptotically stable if and only if all the eigenvalues of the linear operator $£$ have nonnegative real parts. Stability of the equilibrium $\theta^{*}$ has the advantage that the disease inhibition is maintained in its neighborhood, which enables easier control strategies. In particular, the norm of $\theta^{*}$ is a decreasing function of the control $u$.

Proposition 9. The real number $\lambda$ is not an eigenvalue of $£$ if at least one of the following conditions is satisfied:

(i) $\alpha \geq \lambda\left(1-\theta_{1} u\right)$ almost everywhere in $\Omega$ and that inequality is strict on an nonnegligible subset of $\Omega$.

(ii) There exists a real $k \geq 0$ such that for every $\theta \in E$

$$
\int_{\Omega}\langle A(x) \nabla \theta, \nabla \theta\rangle d x \geq k\|\theta\|_{H^{2}(\Omega)},
$$

and

$$
\left(\alpha-\lambda\left(1-\theta_{1} u\right)\right) /\left(1-\theta_{1} u\right)>-k
$$

almost everywhere in $\Omega$. 
Proof: Let $\theta, \varphi \in E$. Then we may compute

$$
\begin{aligned}
& \int_{\Omega}(£ \theta-\lambda \theta) \times \varphi d x \\
& =\int_{\Omega} \varphi \theta\left(\alpha-\lambda\left(1-\theta_{1} u\right)\right) /\left(1-\theta_{1} u\right) d x \\
& \quad \quad-\int_{\Omega} \operatorname{div}(A(x) \nabla \theta) \varphi d x \\
& =\int_{\Omega} \varphi \theta\left(\alpha-\lambda\left(1-\theta_{1} u\right)\right) /\left(1-\theta_{1} u\right)+\langle A(x) \nabla \theta, \nabla \varphi\rangle d x \\
& \equiv p_{1}(\theta, \varphi) .
\end{aligned}
$$

If either of the two conditions of the proposition is satisfied, we may use the Lax-Milgram theorem to obtain the desired result.

Corollary 10. The principal spectrum of $-£$ is contained in $D_{0}^{*} \equiv\left\{\lambda \in \mathbb{C}^{*} ; \operatorname{Re}(\lambda) \leq 0\right\}$.

Proof: From assumption $(H 4), £$ is maximal monotone and $S_{£}$ is a contraction semigroup. Since $S_{£}$ is a contraction semigroup, the resolvant set $\rho(-£)$ of $-£$ contains $\mathbb{R}_{+} \ni\{0\}^{3}$ and $\left\|S_{£}(t)\right\| \leq 1, \forall t \in[0, T]$. Therefore, the spectral radius of $S_{£}(t)$ is less than one. On the other hand $0 \notin \exp \left(t \sigma_{p}(-£)\right) \subseteq \sigma_{p}\left(S_{£}(t)\right) \subseteq$ $\{0\} \bigcup \exp \left(t \sigma_{p}(-£)\right), \forall t \in[0, T]$. Clearly, if $\lambda=\operatorname{Re}(\lambda)+i \operatorname{Im}(\lambda)$ is an element of the principal spectrum of $-£$ then $\exp (\lambda t)$ is an element of the principal spectrum of $S_{£}(t)$ and $|\exp (\lambda t)|=\exp (\operatorname{Re}(\lambda) t)\left\|S_{£}(t)\right\| \leq 1$. It follows that $\operatorname{Re}(\lambda) \leq 0$.

Corollary 11. The equilibrium $\theta^{*}$ is stable. Moreover if all the complex eigenvalues $\lambda$ of the operator $\theta \mapsto \operatorname{div}(A(x) \nabla \theta)$ satisfy $\alpha \geq$ $\left(1-\theta_{1} u\right) \operatorname{Re}(\lambda)$ almost everywhere in $\Omega$, then $\theta^{*}$ asymptotically stable.

\section{Optimal control of the diffusion model}

In the previous section we have seen that the equilibrium of system 140 - 16 was conditionally asymptotically stable. Whether or not the equilibrium $\theta^{*}$ is asymptotically stable, the disease progression shall be contained with respect to some criteria given in terms of costs. The aim

\footnotetext{
${ }^{3}$ See Theorem 3.1 in [25], p8.
}

of this section is to control the system such that the following cost functional is minimized:

$$
J_{T}^{3}(u)=\int_{0}^{T} \int_{\Omega}\left(\theta^{2}+k_{1}(x) u^{2}\right) d x d t+\int_{\Omega} k_{2}(x) \theta^{2}(T, x) d x,
$$

where $k_{1}>0, k_{2} \geq 0$ are bounded penalization terms. The function $k_{1}(x)$ can be interpreted as the cost ratio related to the use of control effort $u$; while $k_{2}$ is the cost ratio related to the magnitude of the final inhibition rate $\theta(T, \cdot)$. In practice, $k_{1}$ reflects the spatial dependence of environmental sensitivity to control means, while $k_{2}$ reflects geographical variations in the cost of the inhibition rate of Colletotrichum at the end of the control period.

In order to establish the optimal control, we will first need to define $U^{K, C}$ as the set of controls $u \in C\left([0, T] ; H^{1}(\Omega ;[0,1])\right)$ such that for every $t, s \in[0, T],\|u(t, \cdot)-u(s, \cdot)\|_{H^{1}(\Omega)} \leq K|t-s|$ and $\|\nabla u(t, \cdot)\|_{L^{2}} \leq C$. For every $K, C \geq 0, U^{K, C}$ is nonempty.

Theorem 12. Let $K, C \geq 0$. Then there is a control $v \in U^{K, C}$ which minimizes the cost $J_{T}^{3}$.

Proof: Since $J_{T}^{3}$ is greater than zero it is bounded below. Let that infinimum be $J^{*}$. There is a sequence $\left(u_{n}\right)_{n \in \mathbb{N}}$ such that the sequence $\left(J_{T}^{3}\left(u_{n}\right)\right)_{n \in \mathbb{N}}$ converges to $J^{*}$. Using definition of $U^{K, C}$ the $\left(u_{n}\right)_{n \in \mathbb{N}}$ is bounded and uniformly equicontinuous on $[0, T]$. By the Ascoli theorem, there is a subsequence $\left(u_{n_{k}}\right)$ which converges to a control $v$. Since the cost function is continuous with respect to $u$ it follows that $J_{T}^{3}(v)=J^{*}$.

We first look the linearized system in the neighborhood of $(\theta, u)=(\varepsilon, 0)$, where $\varepsilon$ depends on $x$. Indeed, this case is of practical significance since the monitoring is assumed to be continuous year-round, and the endemic period corresponds to particular conditions. Thus the outbreak of the disease is "observable" at the moment of onset. The linearized version of 14 is

$\partial \theta / \partial t=\alpha-\alpha \theta-\alpha \varepsilon u \theta_{1}+\operatorname{div}(A(x) \nabla \theta)$, on $] 0, T[\times \Omega$

Note that if $\varepsilon=0$ the linearized system is not controllable. 
D Fotsa et al., Mathematical Modelling and Optimal Control of Anthracnose

Let $£_{1} \theta=-\alpha \theta+\operatorname{div}(A(x) \nabla \theta)$. Equation (23) becomes

$$
\left.\partial \theta / \partial t=£_{1} \theta-\alpha \varepsilon u \theta_{1}+\alpha, \text { on }\right] 0, T[\times \Omega
$$

Theorem 13. The linearized version of $140-16$ has an optimal control in $C\left([0, T] ; L^{2}(\Omega)\right)$ given by

$u(t, \cdot)=\left(1 / k_{1}\right) B P(T-t, \cdot) \theta(t, \cdot)+1 /\left(\varepsilon \theta_{1}\right), t \in[0, T]$

where the linear operator $P$ is solution to the following Riccati equation:

$\dot{P}=£_{1} P+P £_{1}-\left(1 / k_{1}\right) P B^{2} P+I, P(0)=k_{2} I$.

In that equation $I$ is the identity linear operator and $B$ is the linear operator $\alpha \varepsilon \theta_{1} I$.

Proof: (Sketch)

If we set $v=u-1 /\left(\varepsilon \theta_{1}\right)$ then equation 23 becomes

$$
\left.\partial \theta / \partial t=£_{1} \theta-\alpha \varepsilon v \theta_{1}, \text { on }\right] 0, T[\times \Omega .
$$

The rest of the proof is similar to the proof in [29] concerning linear regulators.

If $S_{£_{1}}$ is the contraction semigroup generated by $£_{1}$, then we have $\forall t \in[0, T]$

$$
\begin{gathered}
P(t) f=S_{£_{1}}(t) P(0) S_{£_{1}} f \\
+\int_{0}^{t} S_{£_{1}}(t-s)\left(I-\left(1 / k_{1}\right) P B^{2} P\right) S_{£_{1}}(t-s) f d s
\end{gathered}
$$

Let now consider the nonlinear equation $(14)$. Let $£_{u}$ be the operator $£$ corresponding to control strategy $u$ and let $S_{£_{u}}$ be the contraction semigroup generated by $-£_{u}$. Let

$$
U \equiv\left\{\begin{array}{c}
u \in C\left([0, T] ; H^{1}(\Omega ;[0,1])\right) ; \\
\forall t \in[0, T], S_{£_{u}}(t) \text { is invertible }
\end{array}\right\} .
$$

Some necessary and sufficient conditions for a semigroup of operators to be embedded in a group of operators are given in [25].

Theorem 14. Assume that there is a bounded admissible control $u^{*} \in U$ which minimizes the cost function $J_{T}^{3}$. Let $\tilde{\theta}$ be the absolutely continuous solution of (14) - 16 associated with $u^{*}$. Then $\int_{\Omega}\left(\left(\widetilde{\theta}\left(t_{0}, x\right)\right)^{2}+k_{1}(x)\left(u^{*}\left(t_{0}, x\right)\right)^{2}-p(t) £_{u^{*}} \widetilde{\theta}(t, x)\right) d x$ $\leq \int_{\Omega}\left(\left(\widetilde{\theta}\left(t_{0}, x\right)\right)^{2}+k_{1}(x)\left(u\left(t_{0}, x\right)\right)^{2}-p(t) £_{u} \widetilde{\theta}(t, x)\right) d x$ where $p$ is the absolutely continuous solution on $[0, T]$ of the adjoint state problem

$$
\left\{\begin{array}{l}
\partial p / \partial t=£_{u^{*}} p-2 \widetilde{\theta}, \quad(t, x) \in \mathbb{R}_{+}^{*} \times \Omega \\
\langle A(x) \nabla p, n\rangle=0, \text { on } \mathbb{R}_{+}^{*} \times \partial \Omega \\
p(T)=2 k_{2} \widetilde{\theta}(T, \cdot)
\end{array}\right.
$$

Proof: We give a proof following the maximum principle proof in [29].

For an arbitrary control $w$ and sufficiently small $h \geq 0$, define the needle variation of $u^{*}$ as

$$
u^{h}(t)=\left\{\begin{array}{l}
u^{*}(t), t \in\left[0, t_{0}-h\right] \\
w, t \in] t_{0}-h, t_{0}[ \\
u^{*}(t), t \in\left[t_{0}, T\right]
\end{array}\right.
$$

Let $\theta^{h}$ be the output corresponding to $u^{h}$. Since $u^{*}$ minimizes $J_{T}^{3}, \quad J_{T}^{3}\left(\theta^{h}\right)>J_{T}^{3}\left(\theta^{0}\right)$ and $\partial^{+} J_{T}^{3}\left(\theta^{0}\right) / \partial h>0$.

$$
\begin{aligned}
& \partial^{+} \theta^{0}\left(t_{0}, \cdot\right) / \partial h \\
& =\lim _{h \rightarrow 0^{+}} \frac{1}{h}\left[\theta^{h}\left(t_{0}, \cdot\right)-\theta^{0}\left(t_{0}, \cdot\right)\right] \\
& =\lim _{h \rightarrow 0^{+}} \frac{1}{h} \int_{t_{0}-h}^{t_{0}}\left(£_{u^{0}} \tilde{\theta}(s, \cdot)-£_{u^{h}} \theta^{h}(s, \cdot)\right) d s \\
& =\left(£_{u^{0}}-£_{w}\right) \theta^{0}\left(t_{0}, \cdot\right)
\end{aligned}
$$

Since $v^{h}(t)=u^{*}(t)$ on $\left[t_{0}, T\right]$, for almost $t$ in $\left[t_{0}, T\right], \partial \theta^{h} / \partial t=\alpha-£_{u^{*}} \theta^{h}$.

$$
\begin{aligned}
& \partial\left(\partial^{+} \theta^{0}(t, \cdot) / \partial h\right) / \partial t \\
& =\partial\left(\partial^{+} \theta^{h}(t, \cdot) / \partial h\right) /\left.\partial t\right|_{h=0} \\
& =\partial^{+}\left(\partial \theta^{h}(t, \cdot) / \partial t\right) /\left.\partial h\right|_{h=0} \\
& =\partial^{+}\left(\alpha-£_{u^{*}} \theta^{h}\right) /\left.\partial h\right|_{h=0} \\
& =-\left.£_{u^{*}}\left(\partial^{+} \theta^{h} / \partial h\right)\right|_{h=0} \\
& =-£_{u^{*}}\left(\partial^{+} \theta^{0} / \partial h\right)
\end{aligned}
$$

Therefore,

$\partial^{+} \theta^{0}(t, \cdot) / \partial h=S_{£_{u^{*}}}(t)\left(S_{£_{u^{*}}}\left(t_{0}\right)\right)^{-1}\left(£_{u^{0}}-£_{w}\right) \theta^{0}\left(t_{0}, \cdot\right)$ 
Consequently,

$$
\begin{aligned}
& \partial^{+}\left(\int_{\Omega} k_{2}(x)\left(\theta^{0}(T, x)\right)^{2} d x\right) / \partial h \\
& =2 \int_{\Omega} k_{2}(x) \theta^{0}(T, x) \partial^{+} \theta^{0}(T, x) / \partial h d x \\
& =2 \int_{\Omega} k_{2}(x) \theta^{0}(T, x) S_{£_{u^{*}}}(T)\left(S_{£_{u^{*}}}\left(t_{0}\right)\right)^{-1} \\
& \quad \times\left(£_{u^{0}}-£_{w}\right) \theta^{0}\left(t_{0}, x\right) d x \\
& =2 \int_{\Omega}\left(S_{£_{u^{*}}}\left(t_{0}\right)\right)^{-1} S_{£_{u^{*}}}(T) k_{2}(x) \theta^{0}(T, x) \\
& \quad \times\left(£_{u^{0}}-£_{w}\right) \theta^{0}\left(t_{0}, x\right) d x,
\end{aligned}
$$

and in the same manner

$$
\begin{aligned}
& \partial^{+}\left(\int_{0}^{T} \int_{\Omega}\left(\theta^{0}(t, x)\right)^{2} d x d t\right) / \partial h \\
& =\int_{t_{0}}^{T} \int_{\Omega}\left(\partial^{+}\left(\theta^{0}(t, x)\right)^{2} / \partial h\right) d x d t \\
& =2 \int_{t_{0}}^{T} \int_{\Omega}\left(S_{£_{u^{*}}}\left(t_{0}\right)\right)^{-1} S_{£_{u^{*}}}(t) \theta^{0}(t, x) \\
& \quad \times\left(£_{u^{0}}-£_{w}\right) \theta^{0}\left(t_{0}, x\right) d x d t .
\end{aligned}
$$

Since $\partial^{+} J_{T}^{3}\left(\theta^{0}\right) / \partial h \geq 0$, we have

$$
\begin{aligned}
& \quad 2 \int_{t_{0}}^{T} \int_{\Omega}\left(S_{£_{u^{*}}}\left(t_{0}\right)\right)^{-1} S_{£_{u^{*}}}(t) \theta^{0}(t, x) £_{u^{0}} \theta^{0}\left(t_{0}, x\right) d x d t \\
& \quad+2 \int_{\Omega}\left(S_{£_{u^{*}}}\left(t_{0}\right)\right)^{-1} S_{£_{u^{*}}}(T) k_{2}(x) \theta^{0}(T, x) £_{u^{0}} \theta^{0}\left(t_{0}, x\right) d x \\
& \quad-\int_{\Omega}\left(\left(\widetilde{\theta}\left(t_{0}, x\right)\right)^{2}+k_{1}(x)\left(u^{*}\left(t_{0}, x\right)\right)^{2}\right) d x \\
& \geq 2 \int_{t_{0}}^{T} \int_{\Omega}\left(S_{£_{u^{*}}}\left(t_{0}\right)\right)^{-1} S_{£_{u^{*}}}(t) \theta^{0}(t, x) £_{w} \theta^{0}\left(t_{0}, x\right) d x d t \\
& \quad+2 \int_{\Omega}\left(S_{£_{u^{*}}}\left(t_{0}\right)\right)^{-1} S_{£_{u^{*}}}(T) k_{2}(x) \theta^{0}(T, x) £_{w} \theta^{0}\left(t_{0}, x\right) d x \\
& \quad-\int_{\Omega}\left(\left(\widetilde{\theta}\left(t_{0}, x\right)\right)^{2}+k_{1}(x)\left(w\left(t_{0}, x\right)\right)^{2}\right) d x .
\end{aligned}
$$

Note that $t_{0}$ has been chosen arbitrarily. Let $p$ be the solution of the adjoint state problem (24). Then for every time $t \in[0, T]$,

$\int_{\Omega}\left(\left(\widetilde{\theta}\left(t_{0}, x\right)\right)^{2}+k_{1}(x)\left(u^{*}\left(t_{0}, x\right)\right)^{2}-p(t) £_{u^{*}} \widetilde{\theta}(t, x)\right) d x$

$\leq \int_{\Omega}\left(\left(\widetilde{\theta}\left(t_{0}, x\right)\right)^{2}+k_{1}(x)\left(u\left(t_{0}, x\right)\right)^{2}-p(t) £_{u} \widetilde{\theta}(t, x)\right) d x$.

This theorem shows that the optimal control $u^{*}$ minimizes the following Hamiltonian.

$$
H(\widetilde{\theta}, p, u)=\int_{\Omega}\left(\widetilde{\theta}^{2}+k_{1} u^{2}-p £_{u} \widetilde{\theta}\right) d x
$$

As a result, we have the following necessary condition corresponding to $\partial H / \partial u\left(\widetilde{\theta}, p, u^{*}\right)=0$ :

$$
\int_{\Omega}\left(2 k_{1} u^{*}-\alpha \theta_{1} \widetilde{\theta} p /\left(1-\theta_{1} u^{*}\right)^{2}\right) d x=0 .
$$

Condition (25) is satisfied in particular if

$$
2 k_{1} u^{*}\left(1-\theta_{1} u^{*}\right)^{2}=\alpha \theta_{1} \tilde{\theta} p,
$$

which is analogous to (10) for the within-host model. Then we can adopt the following corresponding strategy

$$
u^{*}(t)= \begin{cases}1 & \text { when } 27 \alpha \theta_{1}^{2} \theta p \geq 8 k_{1}, \\ w_{3}(t) & \text { when } 27 \alpha \theta_{1}^{2} \theta p<8 k_{1},\end{cases}
$$

where $w_{3}(t)$ is the element of $\left[0, \min \left\{\frac{1}{3 \theta_{1}}, 1\right\}\right]$ which is the nearest to the smallest nonnegative solution of the equation 26 .

\section{CONCLUSION}

In this paper two models of anthracnose control have been surveyed. These models both have the general form

$$
\partial \theta / \partial t=f(t, \theta, u)+g(t),
$$

where $f$ is linear in the state $\theta$ but not necessarily in the control $u$. As far as the authors know, this type of control system has not been extensively studied. This may be due to the fact that physical control problems usually do not take this form. The majority of such problems tend to use "additive" controls (see [8], [16] for literature on models). But in models of population dynamics, "mutiplicative" control are often more realistic.

Our first model characterizes the within-host behaviour of the disease. We were able to explicitly calculate an optimal control strategy that effectively reduces the inhibition rate compared to the case where no control is used. In our second model we take into account the spatial spread of the disease by adding a diffusion term. That makes the model more interesting but considerably more difficult to analyze. Moreover, visual evaluation appears more difficult because in this case the state of the system is a function of three spatial variables 
plus time. Although we have provided equations satisfied by the optimal control (for the linearized system), in this paper we do not give a practical method for computing the optimal control. It is possible that adapted gradient methods may be used [2]: this is a subject of ongoing research.

Our models seems quite theoretical, but could be used for practical applications if the needed parameters were provided. Indeed, in the literature [9], [10], [12], [17] there are several attempts to estimate these parameters. The principal advantage of our abstract approach is that it can be used to set automatic means to control the disease which are able to adapt themselves with respect to the host plant and to the parameters values.

Obviously our models can be improved. In particular, several results are based on some conditions of smoothness of parameters, and the control strategy is also very regular. In practice parameters are at most piecewise continuous, and some control strategies are discontinuous. For instance, cultural interventions in the farm are like pulses with respect to a certain calendar. The application of antifungal chemical treatments are also pulses, and the effects of these treatments though continuous are of limited duration. We are currently investigating a more general model that takes into account those irregularities.

\section{ACKNOWLEDGMENTS}

The authors thank Professor Sebastian ANITA for discussion with the first author on modelling diseases spread with diffusion equations. That have been possible through a stay at the University Alexandru Ioan Cuza in Iasi (Romania) in the frame of the Eugen Ionescu scholarship program.

\section{REFERENCES}

[1] Aldila D., Götz T., Soewono E., An optimal control Problem arising from dengue disease transmission mode, Math. Biosci., 242, p9-16, 2013.

[2] Anita S., Arnautu V., Capasso V., An Introduction to Optimal Control Problems in Life Scienes and Economics, Springer Science+Business Media, New York, 2011.
[3] Bella-Manga, Bieyss D., Mouen B., Nyass S., Berry D., Elaboration d'une stratégie de lutte durable et efficace contre L'anthracnose des baies du caféier arabica dans les hautes terres de l'Ouest-Cameroun: bilan des connaissances acquises et perspectives, Colloque Scientifique International sur le Café, 19, Trieste, Mai 2001.

[4] Bieysse D., Bella-Manga D., Mouen B., Ndeumeni J., Roussel J., Fabre V. And Berry D., l'anthracnose des baies une menace potentielle pour La culture mondiale de l'arabica. Plantations, recherche, développement, $\mathrm{Pp}$ 145-152, 2002.

[5] Boisson C., l'anthracnose du caféier, revue de Mycologie, 1960.

[6] Brezis H., Functional Analysis, Sobolev Spaces and Partial Differential Equations, Springer Science+Business Media, New York, 2011.

[7] Chen Z., Nunes M., Silva M., Rodriguez J., Appressorium turgor Pressure of Colletotrichum kahawae might have a role in coffee cuticle Penetration, mycologia, 96(6), pp. 1199-1208, 2004.

[8] Coron J., Control and Nonlinearity, AMS, Mathematical surveys And Monographs, Vol 136, 2007.

[9] Danneberger T., Vargas J., Jones Jr., and Jones A., A Model for weather-based forecasting of anthracnose on annual bluegrass, Phytopathology, Vol. 74, No. 4, pp 448-451, 1984.

[10] Dodd J., Estrada A., Matcham J., Jeffries P., Jeger J., The Effect of climatic factors on Colletotrichum gloeosporioides, causal agent of Mango anthracnose, in the Philippines, Plant Pathology (40), pp 568-575, 1991.

[11] Durand N., Bertrand B., Guyot B., Guiraud J. \& Fontana T., Study on the coffea arabica/Colletotrichum kahawae pathosystem: Impact of a Biological plant protection product, J.Plant Dis.Protect. , 116 (2), pp 78-85, 2009.

[12] Duthie J., Models of the response of foliar parasites to the Combined effects of temperature and duration of wetness, Phytopathology, Vol. 87, No. 11, 1997.

[13] Emvudu Y., Demasse R., Djeudeu D., Optimal control using State-dependent Riccati equation of lost of sight in a tuberculosis model, Comp.Appl.Math., 2013.

[14] Ganesh D., Petitot A., Silva M., Alary R., Lecouls A., Fernandez D., Monitoring of the early molecular resistance responses of coffee (coffea arabica L.) To the rust fungus (hemileia vastatrix) using real-time Quantitative RT-PCR, Plant Science 170, pp 1045-1051, 2006.

[15] Jeffries P., Dodd J., Jeger M., Plumbley R., The biology And the control of Colletotrichum spieces on tropical fruit crops, Plant Pathology (39), pp 343-366, 1990.

[16] Lions J., Contrôlabilité Exacte, Perturbations et Stabilisation de Systèmes Distribués,Tome 1, Masson, Paris, 1988.

[17] Mouen B., Bieysse D., Cilas C., and Notteghem J., Spatio-temporal dynamics of arabica coffee berry disease caused by Colletotrichum kahawae on a plot scale. Plant Dis. 91: 1229-1236, 2007.

[18] Mouen B., Bieysse D., Nyasse S., Notteghem J., and Cilas C., Role of rainfall in the development of cof- 
fee berry disease in coffea arabica Caused by Colletotrichum kahawae, in cameroon, Plant pathology, 2009.

[19] Mouen B., Bieysse D., Njiayouom I., Deumeni J., Cilas C., And Notteghem J.,Effect of cultural practices on the development of arabica Coffee berry disease, caused by Colletotrichum kahawae, Eur J Plant Pathol. 119: 391400, 2007.

[20] Mouen B., Chillet M., Jullien A., Bellaire L., Le gainage Précoce des régimes de bananes améliore la croissance des fruits Et leur état sanitaire vis-à-vis de l'anthracnose (Colletotrichum Musae), fruits, vol. 58, p. 71-81, 2003.

[21] Mouen B., Njiayouom I., Bieysse D., Ndoumbe N., Cilas C., And Notteghem J., Effect of shade on arabica coffee berry disease development: Toward an agroforestry system to reduce disease impact, Phytopathology, Vol. 98, No. 12, 2008.

[22] Muller R., Gestin A., Contribution à la mise au point Des méthodes de lutte contrel'anthracnose des baies du caféier D'arabie (coffea arabica) due à une forme du Colletotrichum coffeanum Noack au Cameroun, café cacao thé, vol. XI (2), 1967.

[23] Muller R., L'évolution de l'anthracnose des baies du Caféier d'arabie (coffea arabica) due à une forme du Colletotrichum Coffeanum Noack au Cameroun, café cacao thé, vol. XIV (2), 1970.

[24] Muller R., La lutte contre l'anthracnose des baies du Caféier arabica due à une souche de Colletotrichum coffeanum au Cameroun, note technique, institut français du café, du cacao et Autres plantes stimulantes, 1971.

[25] Pazy A., Semigroups of Linear Operators and Applications to Partial Differential Equations, vol.44 of Applied Mathematical Sciences, Springer-Verlag, New York, 1983.

[26] Ramos A. And Kamidi R., Determination and significance of the Mutation rate of Colletotrichum coffeanum from benomyl sensitivity to benomyl Tolerance, phytopathology, vol. 72, $\mathrm{N}^{\circ} .2,1982$.

[27] Silva M., Várzea V., Guerra-Guimarães L., Azineira G., Fernandez D., Petitot A., Bertrand B., Lashermes P. And Nicole M., Coffee Resistance to the main diseases: leaf rust and coffee berry disease, Braz. J. Plant Physiol., 18(1): 119-147, 2006.

[28] Wharton P., Dieguez-Uribeondo J., The biology of Colletotrichum acutatum, Anales del Jardín Botánico de Madrid 61(1): 3-22, 2004.

[29] Zabczyk J., Mathematical Control Theory: An Introduction, Birkhäuser, Boston, 1995. 